calculated and analyzed.

Results: A total of 66 patients was eligible for analyses, including 53 patients with usual type and 13 patients with mucinous type of ECA. Patients were classified according to the pattern-based system into pattern A of $21.2 \%$, pattern $\mathrm{B}$ of $22.7 \%$ and pattern $\mathrm{C}$ of $56.1 \%$. Lymph node metastasis were detected $0.0 \%, 6.7 \%$ and $27 \%$ in pattern $\mathrm{A}, \mathrm{B}$ and $\mathrm{C}$, respectively. No recurrence transpired in pattern $\mathrm{A}$ and $\mathrm{B}$. However, 5 patients $(13.5 \%)$ with pattern C recurred during median follow up time of 49.5 months. The 5 -year recurrencefree survival rates were not significantly different (pattern A $100 \%$, B $100 \%$ and C $86.3 \%$ ). On the contrary, the 5 -year overall survival rates were significantly different among the 3 patterns (pattern A 100\%, B 93.3\% and C 72.9\%) ( $\mathrm{p}=0.039)$. The Silva Pattern-based classification is the significant prognostic factors for overall survival (hazard ratio $=5.36$; $95 \%$ confidence interval=1.26-25.76; $\mathrm{p}=0.021$ ).

Conclusion: ECA patients with pattern A, according to the pattern-based classification system, have excellent prognosis without lymph node metastasis. Contrastingly, pattern C tumor associated with the worse overall survival, higher rate of recurrence and lymph node metastasis.

Poster (C16)

Cervical Cancer

https://doi.org/10.3802/jgo.2021.32.\$1.C16

\section{Diagnosis and treatment cervical cancer in early pregnancy: one case report}

\section{Indiarto Wityawan, " Hariyono Winarto}

Oncology Gynecology Division, Department Obstetrics and Gynecology, Medical Faculty of Universitas Indonesia, Jakarta, Indonesia (indiartospog@gmail.com)

Objective: Recently, the incidence of gynecological malignant tumors during pregnancy has increased, mainly due to increased age of pregnant. The most common gynecological malignancy in pregnancy is cervical cancer, accounting for $71.6 \%$, followed by ovarian malignancy, accounting for $7.0 \%$. Cervical cancer during pregnancy fortunately is diagnosed at an early stage. It is important to highlight that the oncologic outcome of cervical cancer during pregnancy is not different from nonpregnant patients, noting that the impact of pregnancy on tumor biology remains unclear. A delay in treatment to achieve fetal viability or to improve outcome of the fetus may be an option for patients with early-stage cervical cancer. Methods: A 38-year-old patient G4P3 presented to Maternity and Neonatology Cipto Mangunkusumo National General Hospital Jakarta with a subsequent diagnosis of a squamous cell moderately differentiated cervical invasive carcinoma confirmed by biopsy. A $2 \times 1.5 \mathrm{~cm}$ tumor on the anterior lip of the cervix spread to left fornix with no evidence of parametrial or vaginal involvement was found on physical examination (International Federation of Gynaecology and Obstetrics 2018 stage IIAI). An abdominal and pelvic magnetic resonance imaging (MRI) was ordered. The study revealed heterogeneity in the anterior aspect of the uterine cervix (dimensions not provided by radiologist). No suspicious lymph nodes or parametrial involvement and no evidence of tumor infiltration in the vagina was noted. Given the results of the MRI and the last menstrual period, an ultrasound was requested which confirmed that the patient was intra uterine 7 weeks pregnant. The case was discussed in the Ethics and Law Committee of the Cipto Mangunkusumo National General Hospital and the forensic and medicolegal departments. Tumor Committee and the following options were entertained. If the patient wished to preserve her pregnancy, our recommendation would be to proceed an option of neoadjuvant chemotherapy after 15 weeks and Cesarean section at 32 weeks followed by radical hysterectomy. We also discussed that if the patient decided to end her pregnancy, then proceed with recommendation of pregnancy termination plus type $\mathrm{C} 1$ radical hysterectomy with ovarian preservation. The decision was made with the patient who decided to undergo a radical hysterectomy with bilateral lymphadenectomy with ovarian preservation and this was performed at 11 weeks' gestation. Histologically consistent with nonkeratinizing, moderately differentiated squamous cell carcinoma. No lymphovascular invasion was found. The deepest invasion was found to reach $5 \mathrm{~mm}$ of the $10 \mathrm{~mm}$ thickness of the cervical wall. There was no tumor mass found at the vaginal incision margin. The right and left parametrial preparations did not reveal a tumor mass. Found 21 right pelvic lymph nodes, all found no tumor mass. Found 15 left pelvic lymph nodes, all found no tumor mass. The patient did not require adjuvant therapy.

Results: Analysis of prognosis of cervical cancer during pregnancy shows no negative impact of pregnancy on the outcome of patients; therefore, pregnancy-preserving management should be considered initially. Pregnancy non-preserving management is chosen in advanced disease (stage IIB or higher or lymph node metastases) or in cases when the patient chooses not to preserve her pregnancy (based on local legislation and usually until the 24th week of gestation). Treatment is thus planned without intention to preserve the fetus. In case of an operable disease (IA2-IB2), a radical hysterectomy with fetus in utero (during the 1st or early-2nd trimester) or after hysterotomy (during the late 2nd trimester) can be performed. In IB3 and higher stages, during first trimester chemoradiation can be applied with the fetus 
in utero (the death of the fetus occurs within few days), while during second trimester a hysterotomy as a first step is advised. This reduces the risk of obstetrical complications (bleeding, rupture of the cervix, diffuse intravascular coagulation) and psychological impact on the patient. Alternatively, before chemoradiotherapy is initiated, feticide can be considered for ethical and psychological reasons.

Conclusion: Radical hysterectomy is the treatment of choice for pregnant patients with early cervical cancer. It affords termination or delivery of the pregnancy at the same time of the treatment is provided. Radical hysterectomy during pregnancy can be safely performed even with the fetus in situ and a subsequent cesarean section.

Poster (C17)

Cervical Cancer

https://doi.org/10.3802/jgo.2021.32.S1.C17

\section{Establishment of a novel cell line from glassy cell carcinoma of the cervix and analysis of genomic landscape}

\author{
Yasunari Ishizuka, ${ }^{1,{ }^{*}}$ Masataka Kudo, ${ }^{1}$ Kanako Hatanaka, ${ }^{2}$ \\ Toraji Amano, ${ }^{3}$ Yutaka Hatanaka, ${ }^{2,3}$, Hidemichi Watari' \\ 'Department of Obstetrics and Gynecology, Hokkaido University, \\ Graduate School of Medicine and Faculty of Medicine, Sapporo, Japan \\ (giants_y_i_203@yahoo.co.jp) \\ ${ }^{2}$ Center for Development of Advanced Diagnostics, Hokkaido University \\ Hospital, Sapporo, Japan \\ ${ }^{3}$ Clinical Research and Medical Innovation Center, Hokkaido University \\ Hospital, Sapporo, Japan
}

Objective: Glassy cell carcinoma (GCC) of the cervix is an extremely rare subtype of cervical cancer with poor prognosis, and the molecular characteristics remain unclear. In this study, we investigated the landscape of genomic alterations of GCC. Methods: We established a novel GCC cell line (HU-6) and analyzed genomic alterations of 160 cancer-related genes (GeneRead Human Comprehensive Cancer Panel, Qiagen) in comparison with the parental tumor and 2 existing GCC cell lines available (TOM-2, HOKUG).

Results: HU-6 was derived from a 31-year-old GCC case, and mimicked the parental tumor both in pathohistological findings and in gene alteration patterns. HPV 18 was detected in HU-6 and TOM-2, while HPV 58 in HOKUG. Common gene alterations in 3 GCC cell lines were detected in 40 loci of 26 cancer-related genes, including alterations of the homologous recombination repair (HRR) pathway (ATM, BRCA2, FANCA, and BRIP1), and the SWI/SNF pathway (PBRM1, EZH2), in addition to single nucleotide variant in Noch1 and TP53, which induces differentiation into squamous epithelium, and a frequent and poor prognosis marker in adenocarcinoma of the cervix, respectively. Only HU-6 had a mutation of KRAS (G12C). Copy number analysis revealed that HU-6 showed amplification of Myc, but HOKUG showed loss of heterozygosity of several genes.

Conclusion: HRR related gene alterations were commonly identified in all three GCC cell lines, suggesting that PARP inhibitors may have antitumor effect for GCC. Moreover, KRAS inhibitors such as AMG510 or ARS1620 may be effective for GCC with KARS mutation (G12C).

Poster (C18)

Cervical Cancer

https://doi.org/10.3802/jgo.2021.32.S1.C18

\section{Evaluation of mobile health applications for cervical cancer in the digital marketplace}

\author{
Jakkapop Kanjak, Naratassapol Likitdee, " \\ Chumnan Kietpeerakool, Amornrat Temtanakitpaisan \\ Khon Kaen University, Khon Kaen, Thailand (polli@kku.ac.th)
}

Objective: To assess the quality of mobile health (mHealth) applications (apps) for cervical cancer using the Mobile App Rating Scale (MARS), APPLICATIONS scoring system, and app rating using specific statements.

Methods: We searched for cervical cancer apps from 2 major mobile operating systems (Google Play Store and Apple iTunes Store) in March 2021. Eligible apps were downloaded and assessed for quality by 2 independent reviewers using multimodal assessment tools.

Results: The overall quality MARS score was $2.61 \pm 0.79$ of 5 . The highest scoring app was "ASCCP Management Guidelines" (3.98). Overall, apps scored highest in the functionality domain, followed by information, engagement, and aesthetics. The mean \pm standard deviation of the APPLICATIONS scoring system was $8.50 \pm 1.71$ of 16 . The highest-rated apps were "ASCCP Management Guidelines," "BSCCP," and "Cervical Cancer Guide." Apps scored highest in the paid subscription and price domain. In contrast, apps scored poorly in the text search, literature used, and subjective presentation domains. Concerning app content, many apps infrequently provide misconceptions about cervical cancer. The apps' rating using specific statements was $7.81 \pm 4.56$ of 16 . Conclusion: Overall, the apps analyzed by the MARS and APPLICATIONS scoring systems demonstrated above-average quality. However, there is a need for improvement in the essential information conveyed by these apps. Moreover, the assessment tools have influenced different app quality rating results, confirming a lack of standardized quality assessment tools for them Health app. 